УДК 621.313.333:62-83

\title{
ОПРЕДЕЛЕНИЕ ПАРАМЕТРОВ СХЕМЫ ЗАМЕЩЕНИЯ ПОГРУЖНОГО ЭЛЕКТРОДВИГАТЕЛЯ НА ОСНОВАНИИ ДАННЫХ ИСПЫТАНИЙ
}

\author{
Ямалиев Виль Узбекович1, \\ rusoil@mail.ru
}
Глазырин Александр Савельевич2,3, asglazyrin@tpu.ru

\section{Буньков Дмитрий Сергеевич 4 , bunkovds@tpu.ru}

Кладиев Сергей Николаевич², kladiev@tpu.ru

\author{
Раков Иван Витальевич2, \\ rakovivan@live.com
}

\author{
Боловин Евгений Владимирович2, \\ orange@tpu.ru
}
Ковалев Владимир Захарович ${ }^{3}$ vz_kovalev@mail.ru
Хамитов Рустам Нуриманович5,6, apple_27@list.ru

1 Уфимский государственный нефтяной технический университет, Россия, 450062, г. Уфа, ул. Космонавтов, 1.

2 Национальный исследовательский Томский политехнический университет, Россия, 634050, г. Томск, пр. Ленина, 30.

3 Югорский государственный университет, Россия, 628012, г. Ханты-Мансийск, ул. Чехова, 16.

4 О०О «Элетим», Россия, 634021, г. Томск, ул. Алтайская, 161А.

5 Омский государственный технический университет, Россия, 644050, г. Омск, пр. Мира, 11

6 Тюменский индустриальный университет, Россия, 625000, г. Тюмень, ул. Володарского, 38.

Одной из основных проблем в процессе эксплуатации скважин, оборудованных установками электрических центробежных насосов, является определение технического состояния погружного электрооборудования и предотвращение его отказов. Среди различных подходов к решению данной задачи можно выделить метод определения технического состояния установки электроцентробежных насосов с использованием полной настраиваемой математической модели установки, включающей модель погружного электродвигателя. Рассмотрен подход к определению и восстановлению необходимых параметров для настройки модели погружного электродвигателя на основании данных, приведённых в протоколе испытаний погружного электродвигателя.

Цель: разработка методики по восстановлению параметров схемы замещения для погружного электродвигателя на основании типовых данных, содержащихся в протоколе приёмо-сдаточных испытаний.

Методы. Предложенный подход основан на совместном использовании настраиваемой динамической модели асинхронного электродвигателя с короткозамкнутым ротором и алгоритмом дифрференциальной эволюции. Фактически решение задачи по определению параметров схемы замещения погружного электродвигателя сводится к решению задачи глобальной оптимизации, т. е. к задаче на поиск глобального (наилучшего) минимума функции.

Результаты. Разработан подход для восстановления параметров схемы замещения погружного электродвигателя, который позволяет устанавливать приемлемые для практики значения параметров схемы замещения для погружного электродвигателя по результатам испьтаний; проверена работоспособность разработанного подхода идентификации с применением методов глобальной оптимизации и математического моделирования погружных асинхронных электродвигателей; полученные оценки являются устойчивыми для всех параметров схемы замещения кроме параметра механической подсистемы.

\section{Ключевые слова:}

Идентисрикация, асинхронная машина, генетические алгоритмы, эвристика, погружной электродвигатель, диффференциальная эволюция, многомерная математическая оптимизация многомерные данные, стохастические алгоритмы.

\section{Описание работы и объекта исследования}

Действующий фонд скважин, оснащённый установками электроцентробежных насосов (УЭЦН), в Российской Федерации составляет более 50 \% и име- ет долговременную тенденцию к росту, что вызвано необходимостью обеспечить максимальные отборы скважин. Для обеспечения контроля эксплуатации УЭЦН выделяется большое количество ресурсов как на сбор, так и на обработку информации. 
Имеющиеся решения контроля действующего фонда скважин фактически являются средством визуализации промысловой информации, например, электротехнические параметры эксплуатации погружного электродвигателя. Назначение таких инструментов в области контроля работы фонда скважин сводится к сигнализации при достижении граничных значений контролируемых параметров. Выбор граничных значений отслеживаемых параметров, как правило, осуществляется на основании расчётов, полученных для каталожных характеристик оборудования. Фактически оборудование, применяемое в нефтегазовой отрасли, в том числе погружные электродвигатели, имеет уникальный набор параметров, обусловленный особенностями технологии изготовления этого оборудования. Использование каталожных характеристик приводит к неточностям при подборе оборудования, а также к невозможности осуществления непрерывного мониторинга параметров эксплуатации фонда скважин, менеджмента энергоэффективности и затрудняет реализацию такого направления, как комплексная интегрированная разработка месторождения. Дополнение имеющихся методов и подходов к подбору и мониторингу оборудования, в основе которых лежат стационарные модели с «примитивной» логикой контроля, методами и способами, базирующимися на эвристических алгоритмах, позволит добиться ряда существенных преимуществ, наиболее очевидным из которых можно назвать повышение точности расчётов при подборе оборудования и мониторинге параметров эксплуатации. Менее явными, но ожидаемыми преимуществами будут служить формализация и использование некоторых лучших практик в области мониторинга процесса эксплуатации, таких как внедрение экспертных систем по работе с глубинно-насосным оборудованием. В конечном итоге достигается реализация полноценной задачи оптимизации для целевых параметров месторождения в целом. Неотъемлемой частью преодоления вышеназванных трудностей является верификация математической модели асинхронного погружного электродвигателя, идентификация параметров которого является сложной и нетривиальной задачей, одно из перспективных решений которой будет описано в данной работе.

Целью данной статьи является разработка методики по восстановлению параметров схемы замещения погружного электродвигателя (ПЭД) на основании типовых данных, содержащихся в протоколе приёмосдаточных испытаний.

Возможность реализовать надёжные и эффективные методы мониторинга и управления УЭЦН связана с разработкой методов определения электромагнитных параметров ПЭД на основе доступных данных. На данный момент известен ряд методов, позволяющих восстанавливать параметры асинхронных электродвигателей [1-12]. Зачастую их реализация для условий добывающей компании практически невозможна, поскольку требует специальных испытательных стендов и нагрузочных устройств, также данные подходы малоприменимы в связи с низкой информативностью типовых протоколов испытаний, предоставляемых ремонтными цехами согласно ГОСТ Р 53472-09 [13].
Предложен подход, позволяющий восстанавливать параметры схем замещения на основании результатов испытаний ПЭД, включая испытание холостого хода и испытание на номинальной нагрузке. На данном этапе исследований авторы предлагают осуществлять идентификацию параметров ПЭД на основании результатов испытаний, полученных в условиях сервисных предприятий. Применение предлагаемого метода к УЭЦН, находящимся в скважинных условиях, затрудняется учётом электромагнитных процессов в погружном кабеле, повышающем трансформаторе и синус-фильтре [7, 8]. Модификация предложенного алгоритма идентификации с учетом данных электромагнитных процессов является предметом дальнейших исследований и в этой работе не рассматривается.

Предложенный подход основан на совместном использовании настраиваемой динамической модели асинхронного электродвигателя с короткозамкнутым ротором (induction motor model, IMM) и алгоритмом дифференциальной эволюции (differential evolution, $D E$ ).

\section{Методология восстановления параметров} схемы замещения ПЭД

Предложенный в работе подход по определению параметров схемы замещения ПЭД сводится к решению задачи глобальной оптимизации, т. е. к задаче на поиск глобального (наилучшего) минимума функции. В данном случае в роли целевой функции выступает функция приспособленности (fitness function), которая, в свою очередь, рассчитывается на основе суммы разностей целевых значений, полученных из протоколов испытаний ПЭД и аналогичных параметров, но определённых при использовании настраиваемой математической модели асинхронного электродвигателя.

Далее рассмотрим основные составляющие предложенной методологии более подробно.

Динамическая модель асинхронного электродвигателя с короткозамкнутым ротором (induction motor model, IMM) является основой разработанного подхода. В (1) приведём систему уравнений, описывающую электромагнитные и электромеханические процессы, происходящие в асинхронном двигателе и представленные в неподвижной системе координат $\alpha, \beta[14,15]$ :

$$
\left\{\begin{array}{l}
\frac{d I_{1 \alpha}}{d t}=\frac{1}{\sigma L_{1}} U_{1 \alpha}-\frac{R_{9}}{\sigma L_{1}} I_{1 \alpha}+\frac{R_{2}^{\prime} L_{m}}{\sigma L_{1} L_{2}^{2}} \Psi_{2 \alpha}+\frac{L_{m}}{\sigma L_{1} L_{2}} z_{p} \omega \Psi_{2 \beta} \\
\frac{d I_{1 \beta}}{d t}=\frac{1}{\sigma L_{1}} U_{1 \beta}-\frac{R_{9}}{\sigma L_{1}} I_{1 \beta}+\frac{R_{2}^{\prime} L_{m}}{\sigma L_{1} L_{2}^{2}} \Psi_{2 \beta}-\frac{L_{m}}{\sigma L_{1} L_{2}} z_{p} \omega \Psi_{2 \alpha} \\
\frac{d \Psi_{2 \alpha}}{d t}=-\frac{R_{2}^{\prime}}{L_{2}} \Psi_{2 \alpha}+\frac{R_{2}^{\prime} L_{m}}{L_{2}} I_{1 \alpha}-z_{p} \omega \Psi_{2 \beta} \\
\frac{d \Psi_{2 \beta}}{d t}=-\frac{R_{2}^{\prime}}{L_{2}} \Psi_{2 \beta}+\frac{R_{2}^{\prime} L_{m}}{L_{2}} i_{1 \beta}+z_{p} \omega \Psi_{2 \alpha} \\
M_{9 \mathrm{M}}=\frac{3}{2} \frac{L_{m}}{L_{2}} z_{p}\left(\Psi_{2 \alpha} I_{1 \beta}-\Psi_{2 \beta} I_{1 \alpha}\right) \\
\frac{d \omega}{d t}=\frac{1}{J}\left(M_{9 \mathrm{~m}}-M_{\mathrm{c}}\right)
\end{array}\right.
$$

где $U_{1 \alpha}=U_{1 m} \sin \left(2 \pi f_{1}\right)=\sqrt{2} U_{1} \sin \left(2 \pi f_{1}\right)$ - синусоидальная по форме составляющая напряжения статора по оси $\alpha$ 
ортогональной неподвижной системы координат $\alpha, \beta, \mathrm{B}$ $U_{1 \beta}=U_{1 m} \cos \left(2 \pi f_{1}\right)=\sqrt{2} U_{1} \cos \left(2 \pi f_{1}\right) \quad$ - косинусоидальная по форме составляющая напряжения статора по оси $\beta$ системы координат $\alpha, \beta, \mathrm{B} ; U_{1 m}=\sqrt{2} \cdot U_{1}-$ амплитудное значение фазного напряжения статорной обмотки, $\mathrm{B} ; U_{1}$ - действующее значение фазного напряжения статорной обмотки, $\mathrm{B} ; f_{1}-$ частота напряжения статора, Гц; $I_{1 \alpha}, \mathrm{I}_{1 \beta}-$ составляющие тока статора в системе координат $\alpha, \beta, \mathrm{A} ; \Psi_{2 \alpha}, \Psi_{2 \beta}$ - составляющие потокосцепления ротора в системе координат $\alpha, \beta$, Вб; $M_{\text {эм }}$ - электромагнитный момент двигателя, $\mathrm{H}^{\cdot} \mathrm{m} ; M_{\mathrm{c}}$ - момент статического сопротивления на валу двигателя, включая собственный момент трения двигателя, Н·м; $\sigma=1-\frac{L_{m}^{2}}{L_{1} \cdot L_{2}}-$ коэффициент рассеяния; $J$ - момент инерции двигателя, кг ${ }^{2}$; $L_{1}=L_{1 \sigma}+L_{b}-$ эквивалентная индуктивность статорной обмотки, Гн; $L_{2}=L_{2 \sigma}^{\prime}+L_{m}-$ эквивалентная индуктивность роторной обмотки, Гн; $L_{m}-$ индуктивность главного контура намагничивания, Гн; $L_{1 \sigma}-$ индуктивность рассеяния обмотки статора, $Г$ н; $L_{2 \sigma}^{\prime}-$ приведенная к статору индуктивность рассеяния обмотки ротора, Гн; $z_{p}$ - число пар полюсов; $R_{1}$ - активное сопротивление статорной обмотки, Ом; $R_{2}^{\prime}-$ приведенное к статору активное сопротивление роторной обмотки, Ом; $R_{1 э}=R_{1}+\frac{R_{2}^{\prime} \cdot L_{m}^{2}}{L_{2}^{2}}-$ эквивалентное сопротивление статора, Ом.

Дифференциальная эволюичя (differential evolution, $D E)[16,17]$ является одной из модификаций стандартного генетического алгоритма (genetic algorithm, $G A)$. Данный алгоритм был предложен сравнительно недавно, в 1995 г., в работе R. Storn и K. Price [16]. Метод дифференциальной эволюции предназначен для нахождения глобального минимума недифференцируемых, нелинейных, мультимодальных (имеющих, возможно, большое число локальных экстремумов) функций от многих переменных [18].

В качестве основных аспектов, повлиявших на выбор данного метода, можно выделить следующие:

- возможность осуществления поиска решения в широком диапазоне значений для функций от большого количества переменных;

- метод прост в реализации и использовании (содержит мало управляющих параметров, требующих подбора) и обеспечивает возможность распараллеливания;

- возможность строго ограничить диапазон поиска для каждой из искомых переменных.

В своей базовой реализации данный алгоритм (рис. 1) управляется двумя параметрами - силой мутации (mutation factor, $F$ ) и вероятностью рекомбинации (crossover probability, $C R$ ). Как и у классического $G A$, в $D E$ начальная популяция формируется как совокупность случайных точек, выбранных из некоторого генерального распределения. На каждой итерации проводится преобразование текущей популяции, согласно установленным правилам. Основным отли- чием $D E$ от $G A$ является следующее: в $D E$ в качестве источника возмущений используется не внешний генератор случайных чисел, а внутренний, реализованный как разность между случайно выбранными векторами текущей популяции. В соответствии с этим, каждая особь в популяции подвергается мутации и рекомбинации, после чего полученные потомки сравниваются с родительскими особями с использованием функции приспособленности и лучшие переходят в следующее поколение, т. е. фактически происходит процесс селекции. Данная особенность обеспечивает быстрое движение по поверхности целевой функции вдоль узких вытянутых «оврагов». Для градиентных же методов в аналогичных условиях характерна колебательная динамика «от стенки к стенке» [19-21].

Рассмотрим параметры, применённые в работе для настройки $D E$ :

- Размерность искомой области (Dimension of parameter vector, $D$ ), соответствующая числу параметров, оцениваемых алгоритмом, выбрана 4 $\left(R_{2}{ }^{\prime}, L_{\sigma}, L_{m}, J\right)$, где $L_{\sigma}=L_{1 \sigma}=L_{2 \sigma}^{\prime}$, Гн. При этом принятое допущение о равенстве индуктивностей рассеяния [17] уменьшает объем требуемых вычислений и ускоряет работу алгоритма.

- Значение размера популяции (population size, NP) принято равным 15 исходя из размерности каждой особи $(D)$.

- Значение силь мутации (mutation factor, $F$ ) задано в диапазоне $[0.0,2.0]$, причём наиболее применимым диапазоном можно считать $[0.5,1.0]$. При подборе необходимо учитывать, что данный параметр в значительной степени влияет на скорость конвергенции и его увеличение позволит расширить «радиус поиска» для преодоления преждевременной сходимости к локальному (нежелательному) экстремуму на многоэкстремальной гиперповерхности целевой функции, но этот фактор исследователю следует выбирать с осторожностью, на основе своего эмпирического опыта, иначе необоснованное расширение этого диапазона негативно скажется на скорости сходимости алгоритма:

$$
\vec{v}_{i, G+1}=\vec{x}_{p, G}+F \cdot\left(\vec{x}_{q, G}-\vec{x}_{r, G}\right),
$$

где $G$ - текущее поколение; $F$ - мутация; $p, q, r$ - случайно выбранные индексы в интервале от 1 до NP так, чтобы $x_{p, G} \neq x_{q, G} \neq x_{r, G}$.

- Значение рекомбинации (crossover probability, CR) задано в диапазоне $[0.0,1.0]$. Увеличение данного параметра позволяет повысить интенсивность мутаций в следующем поколении, при этом необходимо контролировать скорость стабилизации популяции (её вырождение), данный параметр был принят равным 0,7 :

$$
\begin{aligned}
u_{i(j), G+1}= & \left\{\begin{array}{l}
v_{i(j), G+1}, \text { if } r_{i} \leq C R \text { or } j=J_{r} \\
x_{i(j), G}, \text { if } r_{i}>C R \text { or } j \neq J_{r}
\end{array}\right. \\
& \{i, j\}=1,2, \ldots, N P,
\end{aligned}
$$

где $r_{i} \sim U[0,1], J_{r}-$ случайное целое в диапазоне $[1,2, \ldots, D]$, обеспечивающее следующее $\vec{v}_{i, G+1} \neq \vec{x}_{i, G}$. 
- Селекuия (selection) является реализацией оператора выбора, где вектор $\vec{x}_{i, G}$ сравнивается с тестовым вектором $\vec{u}_{i, G+1}$ :

$$
\begin{gathered}
x_{i, G+1}=\left\{\begin{array}{l}
\vec{u}_{i, G+1}, \text { if } f\left(\vec{u}_{i, G+1}\right) \leq f\left(\vec{x}_{i, G}\right) \\
\vec{x}_{i, G}, \text { otherwise }
\end{array}\right. \\
i=1,2, \ldots, N P,
\end{gathered}
$$

где $f(\vec{x})$ - целевая функция, подвергаемая оптимизации.

- Начальное распределение популяции было принято с применением метода latin hypercube sampling (LHS) [22], являющимся статистическим методом для создания псевдослучайной выборки значений параметров из многомерного распределения.

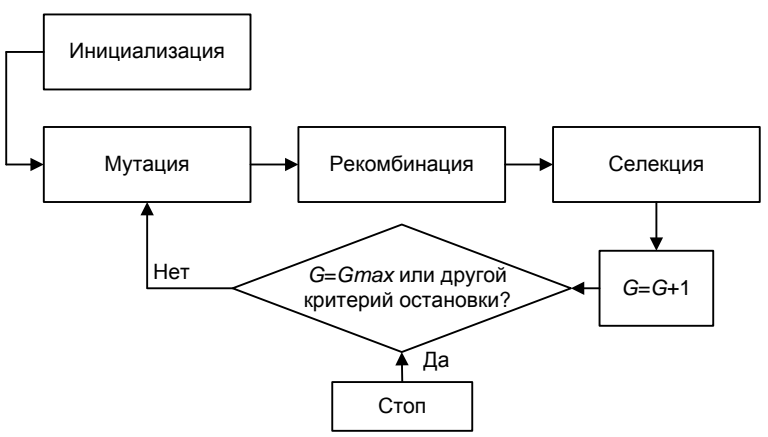

Рис. 1. Схема алгоритма дифференциальной эволюции Fig. 1. Diagram of differential evolution algorithm

Функиия приспособленности (fitness function, FF) это функция оценки, определяющая меру приспособленности полученного решения. В работе была разработана $F F$, устанавливающая связь между целевыми показателями, полученными из протокола испытаний ПЭД (рис. 2), и аналогичными параметрами, полученными из модели IMM.

При проведении испытаний по входному контролю на специализированном предприятии по ремонту УЭЦН испытания ПЭД выполняются согласно ГОСТ Р 53472-09 [13]. В рамках испытаний проводятся следующие опыты:

- опыт короткого замыкания;

- опыт холостого хода;

- испытание при номинальной нагрузке.

По результатам испытаний формируется следующий протокол (рис. 2).

Среди значений, приведённых на рис. 2, были выбраны следующие замеренные параметры:

- «опыт холостого хода» - напряжение разгона, ток холостого хода, сопротивление фаз обмоток статора при постоянном токе в нагретом состоянии;

- «испытания при номинальной нагрузке» - номинальное напряжение, номинальный ток, величина момента проворачивания вала электродвигателя, момент.

Выбор данных параметров основан на следующих доводах и допущениях:
- ряд параметров из протокола испытаний приняты как постоянные для модели IMM (сопротивление фаз обмоток статора при постоянном токе в нагретом состоянии $\left(R_{1}\right)$, число пар полюсов $(z)$, номинальная частота питающего тока $(f))$;

- при стендовых испытаниях асинхронная машина работает при различных режимах, тем самым обеспечивается возможность идентификации её параметров. Существует возможность подбора таких параметров схемы замещения, при которых поведение и значения параметров реального ПЭД и его аналога (настраиваемой математической модели) будут идентичны. Можно предположить, что разность между модельными данными и реальными показателями контролируемых параметров (номинальный ток, ток холостого хода, момент) при установившемся режиме работы ПЭД будет минимальной.

В результате проведённой работы была получена функция оценки $(F F)$, состоящая из четырёх основных частей, достаточных для решения задачи восстановления параметров схемы замещения:

- первая и вторая части выражены в виде функций, описывающих разность между значением замеренного тока, полученного на «испытании при номинальной нагрузке» и «опыте холостого хода», и соответствующих им аналогов в модели $I M M$, причём значение момента нагрузки, прикладываемого к валу двигателя (для испытания при номинальной нагрузке) было рассчитано согласно следующей формуле:

$$
M_{\text {с }}=M_{\text {ном }}=\frac{1000 \cdot P_{\text {ном }}}{2 \cdot \pi \cdot f / z_{p}},
$$

где $P_{\text {ном }}-$ мощность, кВт; $z_{p}$ - число пар полюсов; $f-$ частота питающего тока.

При этом стоит отметить, что фактически значения амплитуды тока были вырождены в единичное значение, рассчитываемое как модуль разности измеренного значения при проведении испытаний и среднего арифметического, полученного из верхней огибающей тока в модели $I M M$ (рис. 3, в):

$$
\begin{gathered}
\operatorname{dist}_{1, i}=\left|I_{\text {nom exper }}-\operatorname{mean}\left(H\left(f_{I}\left(t_{\text {stable }}\right)\right)\right)\right| \\
i=1,2, \ldots, N P,
\end{gathered}
$$

где $f_{I}(t)$ - выходное значение тока по фазе настраиваемой математической модели ПЭД при моно режиме; $H(\cdot)$ - функция для формирования огибающей; mean $(\cdot)-$ среднее арифметическое из функции; $I_{\text {nom exper - номи- }}$ нальное значение тока из протокола испытаний.

- третья и четвертая части функции приспособления учитывают отклонение развиваемого момента (по результатам моделирования) и целевых значений моментов, полученных в процессе натурных испытаний.

Все расчёты проводились с использованием модифицированной версии библиотеки scipy [23-26] и модели IMM собственной реализации. На основании полученных результатов проведена апробация метода определения параметров ПЭД с применением имеющегося протокола испытаний (рис. 2). 


\section{ПРЦЭПУ}

\section{Протокол приемо-сдаточных испытаний №}

\section{ПЭД 45-117 МЭВ5}

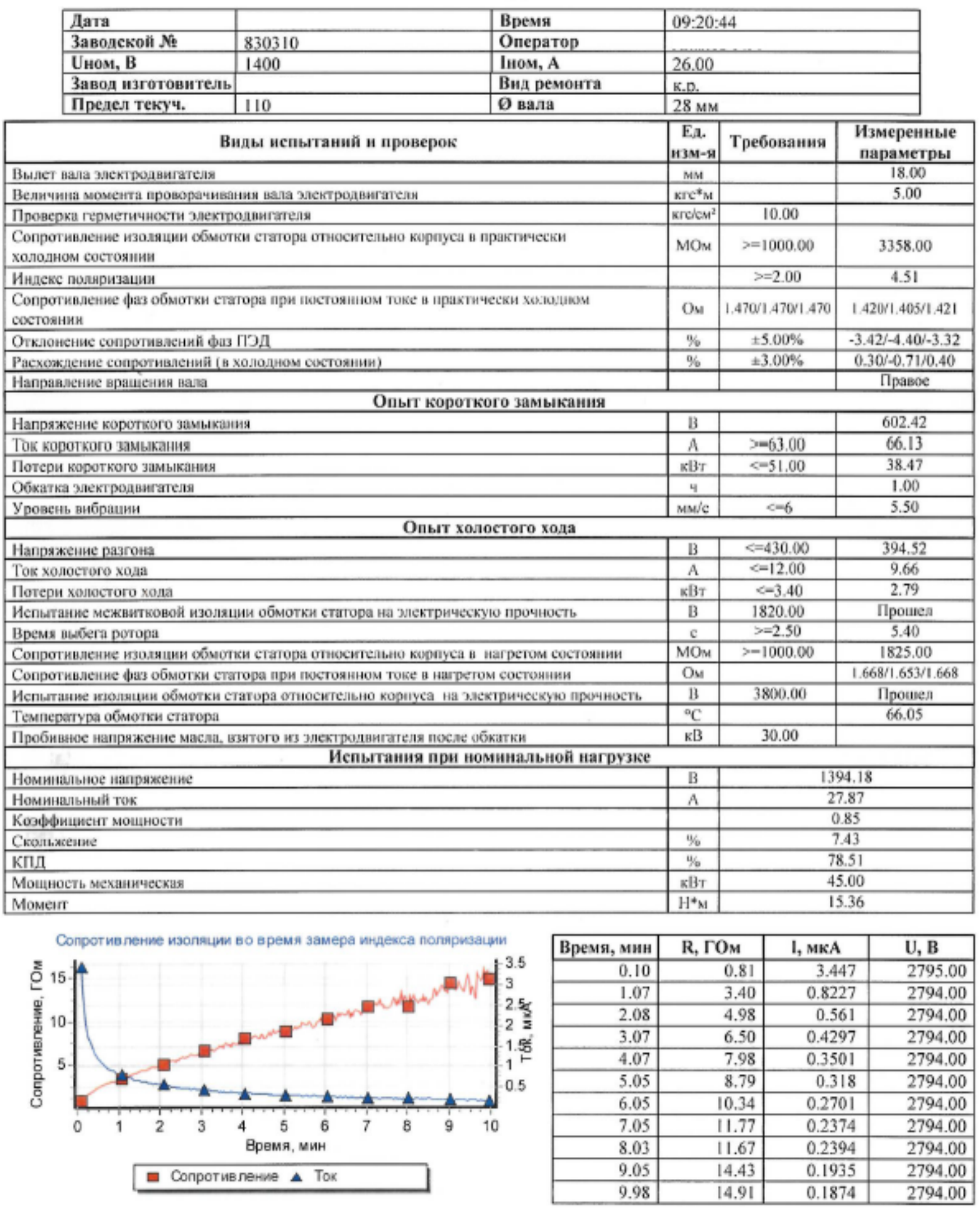

По результатам испытаний, ПЭД признан годным к эксплуатации

Pис. 2. Форма акта испытаний ПЭД

Fig. 2. Form of the submersible induction motor test report 


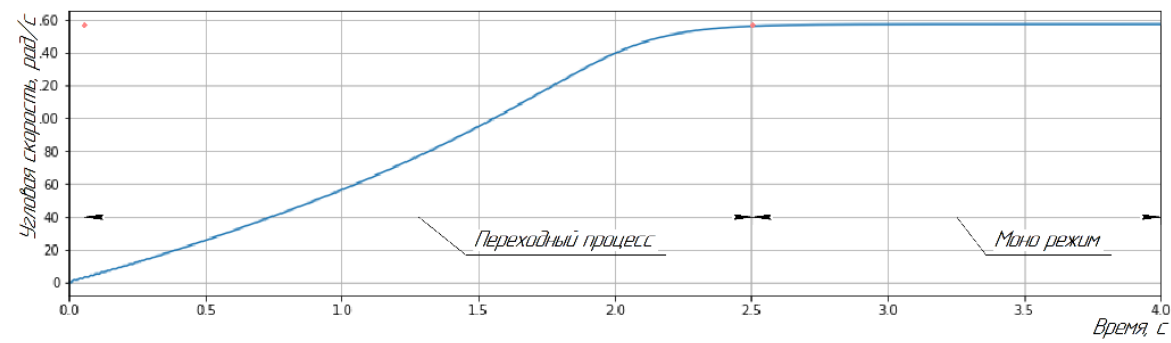

ala

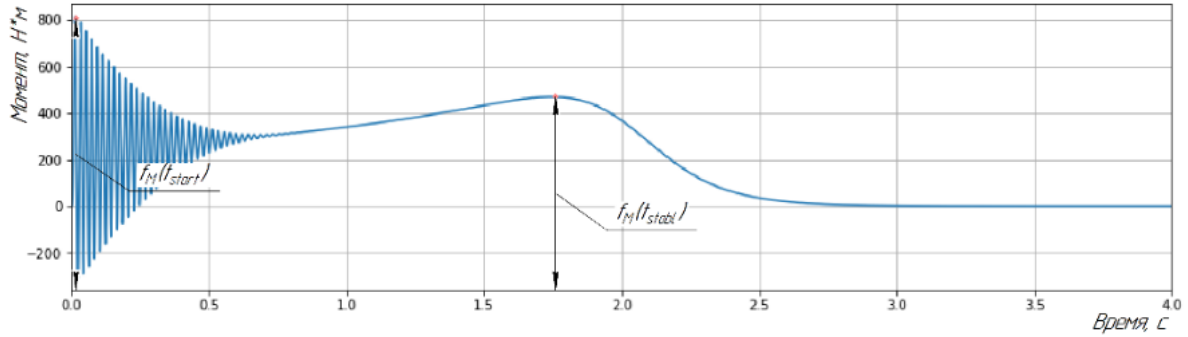

$6 / b$

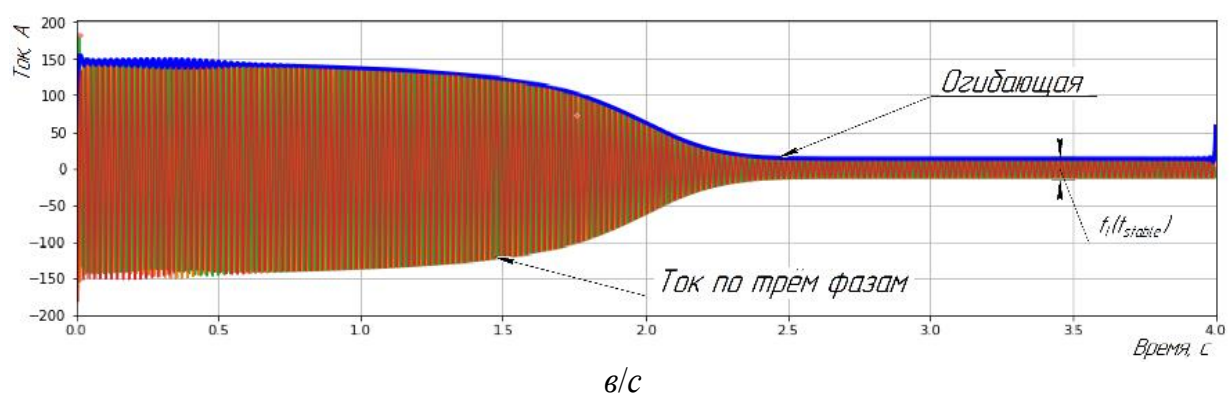

Pис. 3. Пример выходных данных, полученных из модели ПЭД: а) угловая скорость; б) момент; в) фазные токи

Fig. 3. Example of output data obtained from the submersible induction motor model: a) angular velocity; b) moment; c) phase currents

\section{Результаты и обсуждение}

Проверка работоспособности предложенной методики по восстановлению параметров схемы замещения для ПЭД была выполнена для погружного асин- хронного электродвигателя ПЭД 45-117 МЭВ5 (протокол испытаний приведён на рис. 2). В качестве входных данных для расчёта использовались следующие параметры (табл. 1).

Таблица 1. Данные из протокола испытаний для ПЭД 45-117 МЭВ5

Table 1. Data from the test report for the PED 45-117 MEV5

\begin{tabular}{|c|c|c|c|}
\hline $\begin{array}{l}\text { Наименование параметра } \\
\text { Parameter name }\end{array}$ & $\begin{array}{l}\text { Тип испытания } \\
\text { Experiment type }\end{array}$ & $\begin{array}{c}\text { Единицы измере- } \\
\text { ния } \\
\text { Unit measure } \\
\end{array}$ & $\begin{array}{c}\text { Значение } \\
\text { Value }\end{array}$ \\
\hline $\begin{array}{l}\text { Номинальный ток } \\
\text { Rated current }\end{array}$ & $\begin{array}{l}\text { Испытания при номинальной нагрузке } \\
\text { Test at rated load }\end{array}$ & $\mathrm{A} / \mathrm{A}$ & 27,87 \\
\hline $\begin{array}{l}\text { Ток холостого хода } \\
\text { No-load current }\end{array}$ & $\begin{array}{c}\text { Опыт холостого хода } \\
\text { No-load test } \\
\end{array}$ & $\mathrm{A} / \mathrm{A}$ & 9,66 \\
\hline $\begin{array}{l}\text { Напряжение номинальное } \\
\text { Nominal voltage }\end{array}$ & $\begin{array}{c}\text { Испытания при номинальной нагрузке } \\
\text { Test at rated load } \\
\end{array}$ & $\mathrm{B} / \mathrm{V}$ & 1394,18 \\
\hline $\begin{array}{l}\text { Напряжение разгона } \\
\text { Acceleration voltage }\end{array}$ & $\begin{array}{c}\text { Опыт холостого хода } \\
\text { No-load test }\end{array}$ & $\mathrm{B} / \mathrm{V}$ & 394,52 \\
\hline $\begin{array}{l}\text { Мощность механическая } \\
\text { Mechanical power }\end{array}$ & $\begin{array}{c}\text { Испытания при номинальной нагрузке } \\
\text { Test at rated load } \\
\end{array}$ & кВт $/ \mathrm{kW}$ & 45,00 \\
\hline $\begin{array}{l}\text { Сопротивление фаз статора при постоян- } \\
\text { ном токе в нагретом состоянии } \\
\text { Stator phase resistance at constant current in } \\
\text { a heated state }\end{array}$ & $\begin{array}{l}\text { Опыт холостого хода } \\
\text { No-load test }\end{array}$ & Ом/Ohm & 1,653 \\
\hline
\end{tabular}

Общая структура подхода представлена на рис. 4. Настройка подпрограммы-решателя осушествляется в следующем порядке:

- определяются граничные условия для области поиска решения, т. е. для каждого восстанавливае- мого параметра устанавливаются минимальное и максимальное значение -

$R_{2} \in[1,200]^{*} 10^{-2}[\mathrm{OM}], L_{\sigma} \in[1,100] * 10^{-2}\left[\Gamma_{\mathrm{H}}\right]$,

$L_{m} \in[1,1000]^{*} 10^{-2}[\Gamma \mathrm{H}], J \in[1,50]^{*} 10^{-1}\left[\mathrm{\kappa}^{*} \mathrm{M}^{2}\right]$; 
- выполняется начальная инициализация (первая популяция) по методу LHS [17], и устанавливается значение желаемой;

- в качестве целевых параметров принимаются данные, приведённые в табл. 1;
- искомые переменные в системе уравнений (1) принимаются равными 0 (при инициализации);

- временной интервал, для которого разрешается система, определяется исходя из опытных данных. В примере интервал принят от 0 до 25 с.

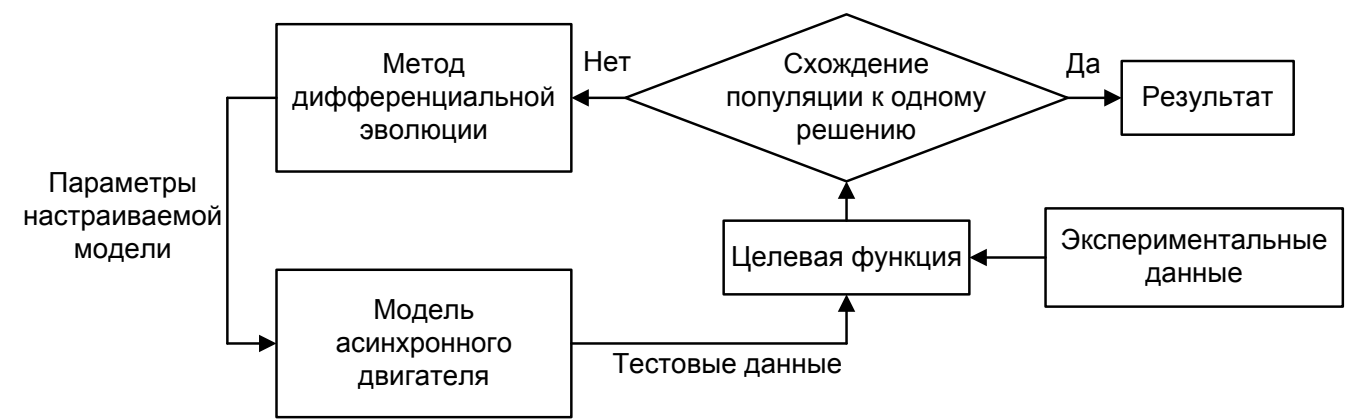

Рис. 4. Блок-схема предложенного подхода

Fig. 4. Block diagram of the proposed approach

В процессе восстановления параметров схемы замещения алгоритм формирует популяцию решений с указанием их близости к целевым параметрам согласно $F F$, на рис. 5 приведён пример популяции решений.

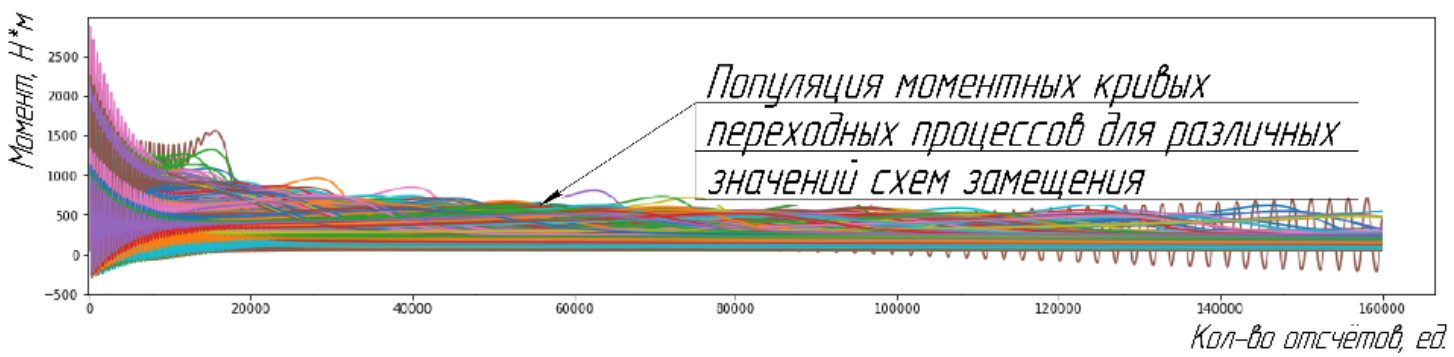

Рис. 5. Популящия моментных кривых, полученных при решении задачи оптимизации

Fig. 5. Population moments curve when solving optimization problems

Более подробно рассмотрим первые три решения с наименьшим значением $F F\left(R_{2}{ }^{\prime}, L_{\sigma}, L_{m}, J\right)$ (табл. 2), являющиеся наиболее близкими к целевым параметрам (табл. 1). Согласно результатам, приведённым в табл. 2, можно сказать о наличии значительной девиации при определении параметра $J$, что свидетельствует о недостаточности исходных данных для его установления, т. е. его влияние на поведение функции приспособления минимально.

Рассмотрим поведение модели асинхронного двигателя при подстановке параметров, соответствую- щих первым двум решениям, полученным с помощью алгоритма дифференциальной эволюции, и количественно опишем отличия в переходных процессах (рис. 6). Фактически отличие полученных решений заключается в моментной кривой $\left(2100 \mathrm{H}^{*} \mathrm{M}-\right.$ Вариант 1 и $3100 \mathrm{H}^{*} \mathrm{M}$ - Вариант 2) и времени разгона (0,6 c - Вариант 1 и 0,2 c - Вариант 2), целевые параметры и потребляемые токи при установившемся режиме близки между собой. Таким образом, в качестве лучшего решения будет принято решение с наименьшим значением $F F$.

Таблица 2. Три лучших решения с минимальными значениями FF

Table 2. Three best solutions with the minimum values of FF

\begin{tabular}{|c|c|c|c|c|c|}
\hline № & $R_{2}{ }^{\prime * 1} 10^{-2}, \mathrm{OM} / \mathrm{Ohm}$ & $L_{\sigma} * 10^{-2}, \Gamma \mathrm{H} / \mathrm{H}$ & $L_{m} * 10^{-2}, \Gamma \mathrm{H} / H$ & $J * 10^{-1}, \mathrm{~K}^{*} \mathrm{M}^{2} / \mathrm{kg}^{*} \mathrm{~m}^{2}$ & $F F\left(R_{2}{ }^{\prime}, L_{\sigma}, L_{m}, J\right)$ \\
\hline 1 & 146,47905067 & 9,72921514 & 220,81600766 & 43,16887615 & 5,481924 \\
\hline 2 & 130,61609658 & 5,03123803 & 224,46268807 & 25,04266715 & 5,758319 \\
\hline 3 & 137,01406269 & 4,12017481 & 203,52997571 & 7,64558847 & 7,104389 \\
\hline
\end{tabular}

Проведённый анализ полученных результатов (рис. 6, табл. 2) позволяет утверждать, что все полученные оценки являются устойчивыми и приемлемыми [27], что говорит о работоспособности разработанной методологии восстановления параметров схемы замещения ПЭД и о принципиальной возможности применения рассмотренного подхода для монито- ринга параметров УЭЦН. Необходимо отметить направления для дальнейших улучшений, позволяющие повысить достоверность получаемых результатов без изменения базовой структуры (рис. 4) предложенного подхода, а именно:

- учёт влияния механической подсистемы ПЭД в математической модели асинхронного двигателя 
либо реализация идентификации параметров электромагнитной и механической подсистем раздельно аналогично работам [7, 8, 25];

- использование в качестве опорной траектории не только модуля результирующего вектора тока статора ПЭД, а также и другие опорные траектории или их сочетание. В качестве перспективных для построения опорных траекторий следует рассматривать сигналы мгновенных реактивной и активной мощностей статора, которые легко получить, применив операции скалярного и векторного произведения векторов тока и напряжения статора в неподвижной ортогональной системе координат $\alpha, \beta[25]$;

- д дополнение $F F$ мерой близости параметров, получаемых при проведении испытаний на короткое замыкание, т. к. данные результаты позволят обеспечить «отсев» решений, не позволяющих достичь максимального момента на валу ПЭД;

- проведение дальнейших исследования по так называемому «предобусловливанию» решаемой задачи, т. е. описание целевой функции таким образом, что её поверхность профиля «улучшается» и увеличиваются расстояния между экстремумами, увеличивается глубина глобального экстремума;

- формализация и реализация механизма формирования границ области поиска на основании опыта и лучших практик в области проектирования и испытания асинхронных машин;

- реализация распределения решений на отдельные потоки для увеличения скорости работы предложенного подхода.
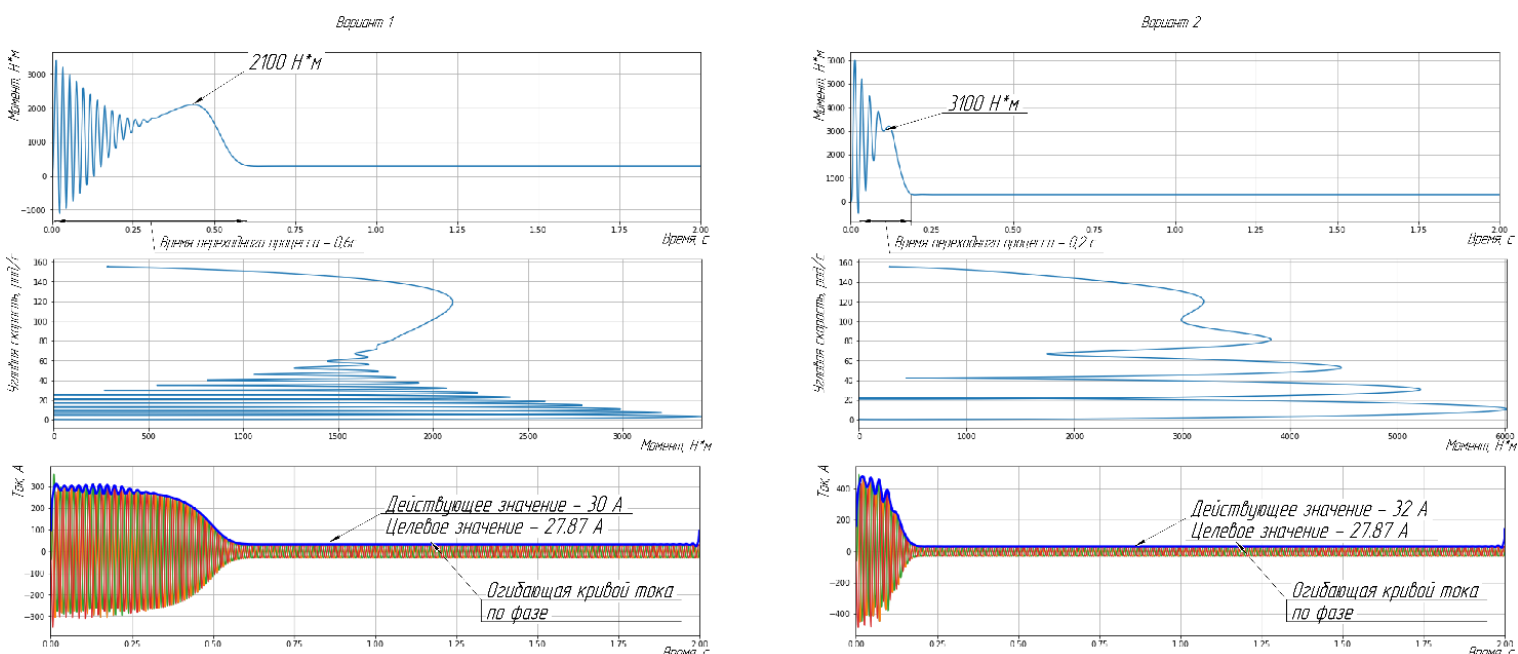

Рис. 6. Сравнение графиков переходных процессов для двух решений согласно табл. 2

Fig. 6. Comparison of the graphs of the input processes for two solutions according to Table 2

\section{Выводы}

1. Разработан подход для восстановления параметров схемы замещения ПЭД, который позволяет устанавливать приемлемые для практики значения параметров схемы замещения для ПЭД по результатам испытаний.

2. Проверена работоспособность разработанного подхода идентификации с применением методов глобальной оптимизации и математического мо-

\section{СПИСОК ЛИТЕРАТУРЫ}

1. Kouhi Ya., Kertzscher J. An online nonlinear identification method for estimation of magnetizing curve and parameters of an induction motor // IFAC - PapersOnLine. - 2017. - V. 50. Iss. 1. - P. 1577-1582.

2. Jirdehi M.A., Rezaei A. Parameters estimation of squirrel-cage induction motors using ANN and ANFIS // Alexandria Engineering Journal. - 2016. - V. 55. - Iss. 1. - P. 357-368.

3. Horen Yo., Strajnikov P., Kuperman A. Simple mechanical parameters identification of induction machine using voltage sensor only // Energy Conversion and Management. - 2015. V. $92 .-$ P. 60-66.

4. Боловин Е.В., Глазырин А.С. Метод идентификации параметров погружных асинхронных электродвигателей установок электроприводных центробежных насосов для добычи нефти // Известия Томского политехнического университета. Инжиниринг георесурсов. - 2017. - Т. 328. - № 1. - С. 123-131 делирования погружных асинхронных электродвигателей. Полученные оценки являются устойчивыми для всех параметров схемы замещения кроме параметра механической подсистемы.

3. Предложенный подход имеет значительный потенциал для своего улучшения как в области повышения точности оценивания параметров настраиваемой модели погружного двигателя, так и в области снижения вычислительных затрат.

5. Tkachuk R.Yu., Glazyrin A.S., Polichshuk V.I. Induction motor drive's parameters identification using genetic algorithms // 7th International Forum on Strategic Technology, IFOST 2012. 2012. - V. 2. - P. 586-589.

6. Применение эвристических алгоритмов в анализе данных для решения задачи диагностирования УЭЦН / Р.И. Валиахметов, В.У. Ямалиев, С.С. Шубин, А.В. Алфёров // Известия Томского политехнического университета. Инжиниринг георесурсов. $-2018 .-$ Т. 329. - № 2. - С. 159-167.

7. Разработка наблюдателя полного порядка с оперативным мониторингом момента сопротивления для погружных асинхронных электродвигателей / А.С. Глазырин, С.Н. Кладиев, К.С. Афанасьев, В.В. Тимошкин, И.Г. Слепнёв, В.И. Полищук, Halász Sándor // Известия Томского политехнического университета. Инжиниринг георесурсов. - 2018. - Т. 329. - № 2. C. $118-126$ 
8. Каширских В.Г. Динамическая идентификация параметров и управление состоянием электродвигателей приводов горных машин: дис. ... д-ра техн. наук. - Кемерово, 2005. - 335 с.

9. Investigation on SVM-Backstepping sensorless control of fivephase open-end winding induction motor based on mode reference adaptive system and parameter estimation / K. Saad, K. Abdellah, H. Ahmed, A. Iqbal // Engineering Science and Technology, an International Journal. - 2019. - V. 22 (4). P. 1013-1026.

10. Kun-Yung Chen, Wen-HaoYang, Rong-Fong Fung. System identification by using RGA with a reduced-order robust observer for an induction motor // Mechatronics. - 2018. - V. 54. - P. 1-15.

11. Adaptive observer design for a class of nonlinear systems. Application to speed sensorless induction motor / M. Farza, M. M'Saad, T. Ménard, A. Ltaief, T. Maatoug // Automatica. 2018. - V. 90. - P. 239-247.

12. Improved identification method of doubly-fed induction generato based on trajectory sensitivity analysis / H. Li, Y. Wu, Q. Li, L. Gong, X. Xie, Z. Chaia, W. Yang // International Journal of Electrical Power \& Energy Systems. - 2021. - V. 125. - P. 1-10.

13. ГОСТ Р 53472-09. Машины электрические вращающиеся. Двигатели асинхронные. Методы испытаний (с Изменениями № 1, 2). - М.: Изд-во стандартов, 2009. - 41 с.

14. Шрейнер Р.Т. Математическое моделирование электроприводов переменного тока с полупроводниковыми преобразователями частоты. - Екатеренбург: УРО РАН, 2000. - 654 с.

15. Bai L., Ma W. Energy-saving Principles and Technologies for Induction Motors. - Hoboken, New Jersey, USA: John Wiley \& Sons Limited, 2019. - 227 p .

16. Storn R., Price K. Differential evolution - a simple and efficient adaptive scheme for global optimization over continuous spaces // Journal of Global Optimization. - 1995. - № 23 (1). - P. 1-12.

17. Storn R., Price K., Lampinen J. Differential evolution: a practical approach to global optimization. - Berlin, Germany: SpringerVerlag, 2005. - $285 \mathrm{p}$.

18. Пантелеев А.В. Метаэвристические алгоритмы поиска глобального экстремума. - М.: МАИ, 2009. - 160 с.
19. Звонков В.Б., Попов А.М. Сравнительное исследование классических методов оптимизации и генетических алгоритмов // Сибирский журнал науки и технологий. - 2013. - № 4 (50). C. 23-27.

20. Афанасьев А.Ю., Макаров В.Г., Ханнанова В.Н. Идентификация параметров трехфазного асинхронного двигателя при изменении начальных значений оценок в широком диапазоне // Известия высших учебных заведений. Проблемы энергетики. -2015 . - № 11. - С. 87-96.

21. Каширских В.Г. Определение параметров асинхронных электродвигателей в процессе их работы // Вестник КузГТУ. 2015. - № 5. - C. 96-102.

22. Shields M.D., Zhang J. The generalization of Latin hypercube sampling // Reliability Engineering \& System Safety. - 2016. V. 148. - P. 96-108.

23. SciPy is a Python-based ecosystem of open-source software for mathematics, science, and engineering // SciPy developers. 2019. URL: https://www.scipy.org/ (дата обращения 18.10.2020).

24. Глазырин А.С. Способы и алгоритмы эффективной оценки переменных состояния и параметров асинхронных двигателей регулируемых электроприводов: дис. ... д-ра тех. наук. Томск, 2016. - $376 \mathrm{c}$.

25. Corrêa F.C., Seixas L.D. Comparison between genetic algorithm and differential evolution tuning a fuzzy controller // Mathematics in Engineering, Science and Aerospace MESA - 2020. - V. 11. № 3. - P. 557-567.

26. A parallel adaptive differential evolution algorithm for electric motor design / M.R. Essaid, L. Idoumghar, J. Lepagnot, M. Brévilliers // 7th International Conference on Metaheuristics and Nature Inspired computing Meta. - Marrakesh, Morocco, 2018. - V. 1 - P. 204-207.

27. Meyer M.C. Probability and mathematical statistics: theory, applications, and practice in R. - Philadelphia, USA: SIAM, 2019. $-707 \mathrm{p}$

Поступила 18.01.2020 2.

\section{Информация об авторах}

Шубин С.C., кандидат технических наук, старший научный сотрудник кафедры машин и оборудования нефтегазовых промыслов, Уфимский государственный нефтяной технический университет.

Ямалиев В.У., доктор технических наук, профессор кафедры машин и оборудования нефтегазовых промыслов, Уфимский государственный нефтяной технический университет.

Глазырин A.C., доктор технических наук, профессор отделения электроэнергетики и электротехники Инженерной школы энергетики Национального исследовательского Томского политехнического университета; профессор Института нефти и газа Югорского государственного университета.

Буньков Д.С., инженер-программист, ООО «Элетим».

Кладиев $\boldsymbol{C . H . , ~ к а н д и д а т ~ т е х н и ч е с к и х ~ н а у к , ~ д о ц е н т ~ о т д е л е н и я ~ э л е к т р о э н е р г е т и к и ~ и ~ э л е к т р о т е х н и к и ~ И н ж е н е р - ~}$ ной школы энергетики Национального исследовательского Томского политехнического университета.

Раков И.В., аспирант отделения электроэнергетики и электротехники Инженерной школы энергетики Национального исследовательского Томского политехнического университета.

Боловин E.B., кандидат технических наук, старший преподаватель отделения электроэнергетики и электротехники Инженерной школы энергетики Национального исследовательского Томского политехнического университета.

Ковалев В.3., доктор технических наук, профессор Института нефти и газа Югорского государственного университета.

Хамитов P.Н., доктор технических наук, доцент, профессор кафедры электрической техники Омского государственного технического университета; профессор кафедры электроэнергетики Тюменского индустриального университета. 
UDC 621.313.333:62-83

\section{ESTIMATION OF SUBMERSIBLE INDUCTION MOTOR EQUIVALENT CIRCUIT PARAMETERS BASED ON TEST DATA}

\author{
Stanislav S. Shubin', \\ rusoil@mail.ru
}

Vil U. Yamaliev', rusoil@mail.ru

\section{Alexander S. Glazyrin',3, asglazyrin@tpu.ru}

\section{Dmitriy S. Bunkov 4 ,} bunkovds@tpu.ru

\section{Sergey N. Kladiev²,} kladiev@tpu.ru

1 Ufa State Petroleum Technological University, 1, Kosmonavtov street, Ufa, 450062, Russia.

2 National Research Tomsk Polytechnic University, 30, Lenin avenue, Tomsk, 634050, Russia.

3 Yugra State University, 16, Chekhov street, Khanty-Mansiysk, 628012, Russia.

4 Eletim Ltd, 161A, Altayskaya street, Tomsk, 634021, Russia.

5 Omsk State Technical University, 11, Mira avenue, Omsk, 644050, Russia.

${ }^{6}$ Tyumen Industrial University, 38 , Volodarsky street, Tyumen, 625000, Russia.

\author{
Ivan V. Rakov², \\ rakovivan@live.com
}

Evgeniy V. Bolovin², orange@tpu.ru

\author{
Vladimir Z. Kovalev ${ }^{3}$, \\ vz_kovalev@mail.ru
}

\section{Rustam N. Khamitov5,6, apple_27@mail.ru}

One of the main problems in operation of wells equipped with installations of electric centrifugal pumps is determining the technical condition of submersible electrical equipment and preventing its failures. Among the various approaches to solving this problem, one can single out a method for determining the technical state of an installation of electric centrifugal pumps using a complete customizable mathemati$\mathrm{cal}$ model of the installation, including a model of a submersible induction motor. This article discusses an approach to determining and restoring the necessary parameters for tuning a submersible induction motor model based on the data given in the submersible induction motor test report.

The main aim of the research is to develop a methodology for restoring the parameters of the equivalent circuit for submersible induction motor on the basis of typical data contained in the acceptance test report.

Methods. The proposed approach is based on the combined use of a tunable dynamic model of a squirrel-cage induction motor and a differential evolution algorithm. The actual solution to determine the parameters of submersible induction motor damage is reduced to solving the global optimization problem, i. e. to the problem of finding the global (best) minimum of a function.

Results. The authors have developed an approach for restoring the parameters of the submersible induction motor equivalent circuit, which allows setting the values of the equivalent circuit parameters for the submersible induction motor, acceptable for practice, according to the test results; the performance of the developed identification approach was tested using global optimization methods and mathematical modeling of submersible induction motors; the obtained estimates are stable for all parameters of the equivalent circuit except for the parameter of the mechanical subsystem.

\section{Key words:}

Identification, induction machine, genetic algorithms, heuristics, submersible induction motor, differential evolution, multivariate mathematical optimization, multivariate data, stochastic algorithms.

\section{REFERENCES}

1. Kouhi Ya., Kertzscher J.. An online nonlinear identification method for estimation of magnetizing curve and parameters of an induction motor. IFAC-PapersOnLine, 2017, vol. 50, Iss. 1, pp. $1577-1582$.

2. Jirdehi M.A., Rezaei A. Parameters estimation of squirrel-cage induction motors using ANN and ANFIS. Alexandria Engineering Journal, 2016, vol. 55, Iss. 1, pp. 357-368.
3. Horen Yo., Strajnikov P., Kuperman A. Simple mechanical parameters identification of induction machine using voltage sensor only. Energy Conversion and Management, 2015, vol. 92, pp. 60-66.

4. Bolovin E.V., Glazyrin A.S. Method for identifying parameters of submersible induction motors of electrical submersible pump units for oil production. Bulletin of the Tomsk Polytechnic University. Geo Assets Engineering, 2017, vol. 328, № 1, pp. 123-131. In Rus.

5. Tkachuk R.Yu., Glazyrin A.S., Polichshuk V.I. Induction motor drive's parameters identification using genetic algorithms. 7th In- 
ternational Forum on Strategic Technology, IFOST 2012, 2012 vol. 2, pp. 586-589.

6. Valiakhmetov R.I., Yamaliev V.U., Shubin S.S., Alferov A.V. Application of heuristic algorithms in analyzing data to solve the problem of detection of electric centrifugal pumping units. Bulletin of the Tomsk Polytechnic University. Geo Assets Engineering, 2018, vol. 329, no. 2, pp. 159-167. In Rus.

7. Glazyrin A.S., Kladiev S.N., Afanasiev K.S., Timoshkin V.V. Slepnev I.G., Polishchuk V.I., Halasz S. Design of full order observer with real time monitoring of load torque for submersible induction motors. Bulletin of the Tomsk Polytechnic University. Geo Assets Engineering, 2018, vol. 329, no. 2, pp. 118-126. In Rus.

8. Kashirskih V.G. Dinamicheskaya identifikatsiva parametrov $i$ upravlenie sostoyaniem elektrodvigateley privodov gornykh mashin. Diss. Dr. Nauk [Dynamic identification of parameters and control of the state of electric motors of drives of mining machines. Dr. Diss]. Kemerovo, 2005. 335 p.

9. Saad K., Abdellah K., Ahmed H., Iqbal A. Investigation on SVMBackstepping sensorless control of five-phase open-end winding induction motor based on model reference adaptive system and parameter estimation. Engineering Science and Technology, an International Journal, 2019, vol. 22 (4), pp. 1013-1026.

10. Kun-Yung Chen, Wen-HaoYang, Rong-Fong Fung. System identification by using RGA with a reduced-order robust observer for an induction motor. Mechatronics, 2018, vol. 54, pp. 1-15.

11. Farza M., M'Saad M., Ménard T., Ltaief A., Maatoug T.. Adaptive observer design for a class of nonlinear systems. Application to speed sensorless induction motor. Automatica, 2018, vol. 90, pp. 239-247.

12. Li H., Wu Y., Li Q., Gong L., Xie X., Chaia Z., Yang W. Improved identification method of doubly-fed induction generator based on trajectory sensitivity analysis. International Journal of Electrical Power \& Energy Systems, 2021, vol. 125, pp. 1-10.

13. GOST R 53472-09. Mashiny electricheskie vrashayushiesya. Dvigateli asinkhronnye. Metody ispytaniay (s Izmeneniyami № 1, 2) [State Standart 7217-87. Rotating machines. Induction motors. Test methods (with Amendments No. 1, 2)]. Moscow, StandardInform Publ., 2009. 41 p.

14. Sreyner R.T. Matematicheskoe modelirovanie elektroprivodov peremennogo toka s poluprovodnikovymi preobrazovatelyami chastoty [Mathematical modeling of AC electric drives with semiconductor frequency converters]. Ekaterenburg, URO RAN Publ., 2000. 654 p.

15. Bai L., Ma W. Energy-saving principles and technologies for induction motors. Hoboken, New Jersey, USA, John Wiley \& Sons Limited, 2019. 227 p .

16. Storn R., Price K. Differential evolution - a simple and efficient adaptive scheme for global optimization over continuous spaces. Journal of Global Optimization, 1995, no. 23 (1), pp. 1-12.
17. Storn R., Price K., Lampinen J. Differential evolution: a practical approach to global optimization. Berlin, Germany, SpringerVerlag, 2005. $285 \mathrm{p}$.

18. Panteleev A.V. Metaevresticheskie algoritmy poiska globalnogo ekstremuma [Metaheuristic algorithms for finding a global extremum]. Moscow, Moscow Aviation Institute Publ., 2009. 160 p.

19. Zvonkov V.B., Popov A.M. Sravnitelnoe issledovanie klassicheskikh metodov optimizatsii i geneticheskikh algoritmov [Comparative study of classical optimization methods and genetic algorithms]. Siberian journal of science and technology, 2013, no. 4 (50), pp. 23-27.

20. Afanasiev A.Yu., Makarov V.G., Khannanova V.N. Identifikatsiya parametrov trekhfaznogo asinkhronnogo dvigatelya pri izmerenii nachalnykh znacheniy otsenok $\mathrm{v}$ shirokom diapozone [Identification of the parameters of a three-phase asynchronous motor when changing the initial values of the estimates in a wide range]. Power engineering: research, equipment, technology, 2015, no. 11, pp. 87-96.

21. Kashirskikh V.G. Opredelenie parametrov asinhronnykh elektrodvigateley v protsesse ikh raboty [Induction motors parameters estimation during their operation]. Vestnik KuzGTU, 2015, no. 5 , pp. $96-102$.

22. Shields M.D., Zhang J. The generalization of Latin hypercube sampling. Reliability Engineering \& System Safety, 2016, vol. 148, pp. 96-108.

23. SciPy is a Python-based ecosystem of open-source software for mathematics, science, and engineering. SciPy developers. 2019. Available at: https://www.scipy.org/ (accessed 18 October 2020).

24. Glazyrin A.S. Sposoby i algoritmy effectivnoy otsenki peremennykh sostoyaniva i parametrov asinkhronnykh dvigateley reguliruemykh electroprivodov. Diss. Dr. nauk [Methods and algorithms for effective estimation of the state and parameters of induction motors of variable speed drives. Dr. Diss.]. Tomsk, 2016. 376 p.

25. Corrêa F.C., Seixas L.D. Comparison between genetic algorithm and differential evolution tuning a fuzzy controller. Mathematics in Engineering, Science and Aerospace MESA, 2020, vol. 11, no. 3, pp. 557-567.

26. Essaid M., Idoumghar L., Lepagnot J., Brévilliers M. A parallel adaptive differential evolution algorithm for electric motor design. $7^{\text {th }}$ International Conference on Metaheuristics and Nature Inspired computing Meta. Marrakesh, Morocco, 2018. Vol. 1, pp. 204-207.

27. Meyer M.C. Probability and mathematical statistics: theory, applications, and practice in R. Philadelphia, USA, SIAM, 2019. $707 \mathrm{p}$.

Received: 18 January 2020.

\section{Information about the authors}

Stanislav S. Shubin, Cand. Sc., senior research scientist, Ufa State Petroleum Technological University.

Vil U. Yamaliev, Dr. Sc., professor, Ufa State Petroleum Technological University.

Alexander S. Glazyrin, Dr. Sc., professor, National Research Tomsk Polytechnic University; professor, Yugra State University.

Dmitriy S. Bunkov, software engineer, Eletim Ltd.

Sergey N. Kladiev, Cand. Sc., associate professor, National Research Tomsk Polytechnic University.

Ivan V. Rakov, postgraduate student, National Research Tomsk Polytechnic University.

Evgeniy V. Bolovin, Cand. Sc, senior lecture, National Research Tomsk Polytechnic University.

Vladimir Z. Kovalev, Dr. Sc., professor, Yugra State University.

Rustam N. Khamitov, Dr. Sc., professor, Omsk State Technical University; professor, Tyumen Industrial University. 